\title{
Atenção à saúde de imigrantes haitianos em diferentes países na atualidade: revisão integrativa de literatura
}

\author{
Health care for Haitian immigrants in different countries nowadays: an \\ integrative literature review
}

\section{Atención de salud para inmigrantes haitianos en diferentes países hoy: una revisión integradora de literatura}

Thalyta Nogueira Araújo ${ }^{1, a}$

thalytanaraujo@outlook.com | https://orcid.org/o0oo-0003-4417-0741

Juliana Dias Reis Pessalacia ${ }^{1, b}$

juliana@pessalacia.com | https://orcid.org/0000-0003-0468-2283

Priscila Balderrama ${ }^{1, c}$

priscilabalderrama@gmail.com | https://orcid.org/oooo-0003-1979-611X

Aridiane Alves Ribeiro ${ }^{2, d}$

aridianeribeiro@gmail.com | https://orcid.org/0000-0003-2702-9332

Fernando Ribeiro dos Santos ${ }^{1, e}$

fernanndoribeiro@hotmail.com | https://orcid.org/o00o-0002-8913-5205

\footnotetext{
${ }^{1}$ Universidade Federal de Mato Grosso do Sul, Campus de Três Lagoas. Três Lagoas, MS, Brasil.

2 Universidade Federal de Goiás, Campus de Jataí. Jataí, GO, Brasil.

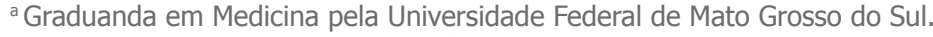

b Doutorado em Enfermagem Psiquiátrica pela Universidade de São Paulo.

c Doutorado em Enfermagem Fundamental pela Universidade de São Paulo.

${ }^{\text {d }}$ Doutorado em Enfermagem pela Universidade de São Paulo.

e Graduando em Medicina pela Universidade Federal de Mato Grosso do Sul.
}

\section{RESUMO}

Com a globalização, os fluxos migratórios intensificaram-se e as razões financeiras são umas das principais impulsionadoras de tais eventos. As populações migrantes têm diferentes necessidades, sendo o acesso à saúde algo primordial para garantir a qualidade de vida. Assim, este estudo objetivou analisar a produção científica sobre atenção à saúde de imigrantes internacionais haitianos em diferentes contextos e países, buscando compreender quais são os desafios e as perspectivas para a atenção à saúde dessa população nos contextos investigados. Trata-se de uma Revisão Integrativa de Literatura, realizada a partir de pesquisas nas bases Sistema Online de Busca e Análise de Literatura Médica (Medline/PubMed) e Scientific Electronic Library Online (SciELO), com os descritores padronizados 'Health Care' ('Public Health'), 'Haiti' e 'Emigrants and Immigrants', combinados por meio do operador booleano AND. Selecionaram-se artigos publicados no período de 2015 a 2019 que fossem em português, inglês ou espanhol e que tratassem desse tema. A partir da análise dos artigos, identificaram-se três categorias de análise temática: Impactos das regulamentações imigratórias na integração dos imigrantes haitianos; Competência intercultural para a atenção à saúde de imigrantes haitianos; e Condições de saúde e acesso a serviços por imigrantes haitianos. Destaca-se a importância da inclusão de aspectos políticos interculturais nas políticas de saúde voltadas à atenção à saúde do imigrante.

Palavras-chave: Emigração e Imigração; Competência cultural; Assistência à saúde culturalmente competente; Haiti; Políticas Públicas. 


\section{ABSTRACT}

With globalization, migration flows have intensified and financial reasons are one of the main drivers of these events. Migrant populations have different needs, and access to health is essential to ensure their quality of life. Accordingly, this study aimed to analyze the scientific production on health care of international Haitian immigrants in different contexts and countries, seeking to understand what are the challenges and perspectives for the health care of this population in the investigated contexts. This is an Integrative Literature Review, performed from searches in the Medical Literature Analysis and Retrieval System Online (Medline/PubMed) and Scientific Electronic Library Online (SciELO), with the standardized descriptors 'Health Care' ('Public Health'), 'Haiti' and 'Emigrants and Immigrants', combined through the Boolean operator AND. We selected articles published in the period 2015-2019, in Portuguese, English or Spanish, that dealt with this theme. From the analysis of the articles, we identified three categories of thematic analysis: Impacts of immigration regulations on the integration of Haitian immigrants; Intercultural competency for the health care for Haitian immigrants; and Health conditions and access to services by Haitian immigrants. We should highlight the importance of including intercultural political aspects in health policies focused on the health care for immigrants.

Keywords: Emigration and Immigration; Cultural competency; Culturally competent care; Haiti; Public Policies.

\section{RESUMEN}

Con la globalización, los flujos migratorios se han intensificado y las razones financieras son uno de los principales impulsores de tales eventos. Las poblaciones migrantes tienen diferentes necesidades, siendo el acceso a la salud fundamental para garantizar la calidad de vida. Así, este estudio tuvo como objetivo analizar la producción científica en salud de inmigrantes haitianos internacionales en diferentes contextos y países, buscando comprender cuáles son los desafíos y perspectivas para la atención de la salud de esta población en los contextos investigados. Se trata de una Revisión Integrativa de Literatura, realizada a partir de la investigación del Sistema de Análisis y Búsqueda de Literatura Médica en Línea (Medline / PubMed) y Biblioteca Electrónica Científica en Línea (SciELO), con los descriptores estandarizados 'Health Care' ('Salud Pública'), 'Haití' y 'Emigrantes e inmigrantes', combinados a través del operador booleano AND. Se seleccionaron artículos publicados en el período de 2015 a 2019 que estuvieran en portugués, inglés o español y abordaran este tema. Del análisis de los artículos se identificaron tres categorías de análisis temático: Impactos de las regulaciones migratorias en la integración de inmigrantes haitianos; Competencia intercultural para la atención de la salud de los inmigrantes haitianos; y Condiciones de salud y acceso a servicios de inmigrantes haitianos. Se destaca la importancia de incluir aspectos políticos interculturales en las políticas de salud dirigidas a la atención de la salud de los inmigrantes.

Palabras clave: Emigración e Inmigración; Competencia cultural; Asistencia sanitaria culturalmente competente; Haití; Políticas Públicas. 
Contribuição dos autores:

Concepção e desenho do estudo: Thalyta Nogueira Araújo; Juliana Dias Reis Pessalacia; Priscila Balderrama.

Aquisição, análise ou interpretação dos dados: Thalyta Nogueira Araújo; Juliana Dias Reis Pessalacia; Priscila Balderrama. Redação do manuscrito: Thalyta Nogueira Araújo; Juliana Dias Reis Pessalacia; Priscila Balderrama; Aridiane Alves Ribeiro; Fernando Ribeiro dos Santos.

Revisão crítica do conteúdo intelectual: Thalyta Nogueira Araújo; Juliana Dias Reis Pessalacia; Priscila Balderrama; Aridiane Alves Ribeiro; Fernando Ribeiro dos Santos.

Declaração de conflito de interesses: não há.

Fontes de financiamento: Apoio da Universidade Federal de Mato Grosso do Sul (UFMS), através do Programa de Bolsa de Iniciação Científica (PIBIC), vinculado ao Conselho Nacional de Desenvolvimento Científico e Tecnológico (CNPq), Edital UFMS PIBIC/PIBIC-AF n 123, de 17 de julho de 2018.

Considerações éticas: não há.

Agradecimentos/Contribuições adicionais: não há.

Histórico do artigo: submetido: 07 abr. 2020 | aceito: 12 ago. 2020 | publicado: 22 mar. 2021.

Apresentação anterior: Artigo vinculado ao projeto "Acesso aos serviços de saúde por imigrantes internacionais no interior do Mato Grosso do Sul".

Licença CC BY-NC atribuição não comercial. Com essa licença é permitido acessar, baixar (download), copiar, imprimir, compartilhar, reutilizar e distribuir os artigos, desde que para uso não comercial e com a citação da fonte, conferindo os devidos créditos de autoria e menção à Reciis. Nesses casos, nenhuma permissão é necessária por parte dos autores ou dos editores.

\section{INTRODUÇÃO}

Os estudos sobre migração e os impactos da questão intercultural iniciaram-se no começo do século XX, em decorrência da crescente mobilidade populacional da Europa, em situação de crise e guerra (ESCUDEIRO, 2019).

O fortalecimento da industrialização, o crescimento da urbanização, os problemas sociais e econômicos decorrentes desses processos e, mais recentemente, as disputas geopolíticas e a globalização impulsionaram o crescimento do deslocamento populacional. Atualmente, os conflitos e as questões financeiras tornaramse as causas mais comuns dos fluxos migratórios (HAYWARD; HARDING; MCSHANE; TANNER, 2018).

Migração pode ser entendida como um processo mundial que ocorre de modo concomitante em diferentes regiões e sob várias facetas. Engloba basicamente dois movimentos: a emigração referente à saída da pessoa de um local e a imigração relacionada à chegada do indivíduo em outro lugar (BRZOZOWSKI, 2012; URQUIZA; RIBEIRO, 2018).

Tal fenômeno caracteriza-se por uma maior integração econômica, gerenciada no âmbito do liberalismo, a qual propicia que o horizonte de migração passe a ser 'o mundo'. Entretanto, os resultados dessa globalização implicam o aumento das desigualdades internacionais, passando a existir restrições de deslocamentos entre alguns países, pois, apesar de os fluxos migratórios serem intensos, as fronteiras já não são mais livres. Assim, investe-se em políticas voltadas à atração de imigrantes, mas considerando-se o tipo de imigrante que se deseja, ou seja, nos atributos que irão favorecer a economia do país (OLIVEIRA; SILVA; OLIVEIRA, 2019).

No Canadá, por exemplo, oitavo país no mundo em número de estrangeiros, facilita-se a entrada de pessoas jovens, com fluência na língua nativa e qualificadas. Já os Estados Unidos da América (EUA) optam 
por imigrantes qualificados que possam se inserir no mercado de trabalho nas áreas em que o país possui maior necessidade, fornecendo vistos temporários e permanentes.

Contudo, tem crescido nos países um grupo específico de imigrantes, conhecido como refugiados, por exemplo, o caso dos venezuelanos e haitianos, em que alguns entram nos países de forma legal e outros, sem autorização, com destaque para o aumento de imigrantes refugiados no Brasil. Os haitianos, após o terremoto de 2010, tiveram o ápice no número de solicitações em 2014, alcançando mais de 16 mil pedidos. Já a chegada de venezuelanos é mais recente, decorrente da grave crise econômica do país, com 4 mil pedidos de refúgio em 2016 e quase 18 mil em 2017 (OLIVEIRA; SILVA; OLIVEIRA, 2019).

Portanto, os fluxos imigratórios desse grupo têm se intensificado e tal processo apresenta implicações para as condições de acesso à saúde, podendo gerar conflitos entre as populações nativas e os imigrantes devido a particularidades dos indivíduos envolvidos e do sistema de saúde do país (GUERRA; VENTURA, 2017). Quanto a esses conflitos, exemplifica-se o caso dos imigrantes haitianos de uma cidade no Amazonas, Brasil, onde foram encontradas uma precariedade das condições de acolhimento dos mesmos, a exploração nos valores cobrados pelos aluguéis em residências frequentemente insalubres, além de uma espécie de 'medo sanitário' fomentado pelos próprios moradores do município (GRANADA; CARRENO; RAMOS; RAMOS, 2017). Devido a isso, é necessário que tais particularidades sejam levadas em consideração na formulação de políticas públicas de saúde nos países (GUERRA; VENTURA, 2017).

Acesso à saúde é um termo complexo de conceituação e variável de acordo com os autores. Entendemos acesso como a acessibilidade e o uso dos serviços de saúde. É influenciado por aspectos funcionais e estruturais da organização dos estabelecimentos de saúde. O grau de acesso também é determinado pela qualidade da assistência prestada, elemento que, por vezes, pode ser a principal barreira de acesso por imigrantes internacionais, como diferenças linguísticas e culturais (BISPO et al., 2020).

As barreiras ao uso dos serviços de saúde por populações migrantes também estão relacionadas às suas necessidades particulares, bem como preferências e expectativas de cuidados de saúde (KLEIN; KNESEBECK, 2018). Nos Estados Unidos da América (EUA) e Canadá, por exemplo, mostram-se como barreiras de acesso à saúde por imigrantes: comunicação/idioma, falta de conhecimento de como se introduzir no serviço de saúde, antagonismos culturais e falta de seguro-saúde. Já no contexto brasileiro, as barreiras culturais mostram-se relevantes, devendo-se considerar para um melhor acesso à saúde as particularidades históricas e culturais dos imigrantes e sua implicação na saúde (FAQUETI; GRISOTTI; RISSON, 2017).

O Haiti tem se destacado como um dos países com maior fluxo migratório em razão do contexto após o terremoto de 2010, o qual o classificou como a nação mais pobre das Américas e do Hemisfério Ocidental, com um Produto Interno Bruto (PIB) per capita de 2016 de US\$820, uma das expectativas de vida mais baixas (64 anos) e uma taxa de alfabetização das mais baixas do mundo (76\%), que o classifica como uma nação com baixo desenvolvimento humano (SÁNCHEZ P, 2018).

Embora a organização dos serviços de saúde e dos sistemas de saúde varie de um país para outro, os migrantes e as minorias étnicas correm maiores riscos de sofrer as consequências de uma atenção à saúde não qualificada, quando comparados com o restante da população (DAUVRIN; LORANT, 2015). Tais consequências evidenciam-se em maiores taxas de desnutrição, problemas respiratórios e intestinais, diarreia e doenças infecciosas, além de sofrerem discriminação, situações de violência e precariedade socioeconômica (GRANADA; CARRENO; RAMOS; RAMOS, 2017).

No Brasil, a Constituição Federal e o Sistema Único de Saúde (SUS) garantem acesso universal à saúde, não fazendo acepção. Assim, constata-se que os imigrantes possuem os mesmos direitos da população nativa. Os princípios que regem o SUS (universalidade, equidade e integralidade) devem orientar o acolhimento 
de todos os usuários, inclusive imigrantes a fim de assegurar atendimento centrado na comunidade, considerando suas particularidades e vulnerabilidades (SANTOS, 2016).

Apesar disso, frequentemente os profissionais de saúde não se encontram preparados para atender a população imigrante no país, dessa forma muitas demandas desse grupo ficam descobertas. As situações precárias de trabalho e moradia com acesso limitado aos serviços de saúde os colocam em um grupo de vulnerabilidade. Nessa conjuntura, seus direitos não são cumpridos, o que aumenta os riscos para saúde dessa população (RISSON; MATSUE; LIMA, 2018).

Diante do intenso fluxo migratório de haitianos e das barreiras de acesso aos serviços de saúde por imigrantes, objetiva-se, a partir desta revisão, analisar a produção científica sobre atenção à saúde de imigrantes internacionais haitianos em diferentes contextos e países, buscando-se compreender quais são os desafios e as perspectivas para a atenção à saúde dessa população nos contextos investigados.

\section{MÉTODO}

Trata-se de uma Revisão Integrativa de Literatura (RIL), que visa responder à seguinte questão: quais são os estudos que abordam a temática da atenção à saúde de imigrantes haitianos em diferentes contextos e países?

A RIL consiste em uma abordagem ampla de revisão de literatura, a qual permite a inclusão de estudos experimentais e não experimentais para uma compreensão completa do fenômeno analisado. Pode contemplar dados da literatura teórica e empírica e diferentes propósitos com o objetivo de gerar um panorama consistente e compreensível de conceitos, teorias ou, neste caso, iluminar problemas de saúde relevantes (SOUZA; SILVA; CARVALHO, 2010).

Para tal, percorreram-se as seguintes etapas: estabelecimento da hipótese e objetivos da revisão integrativa; estabelecimento de critérios de inclusão e exclusão de artigos (seleção da amostra); definição das informações a serem extraídas dos artigos selecionados; análise dos resultados; discussão e apresentação dos resultados; e apresentação da revisão (MENDES; SILVEIRA; GALVÃO, 2008).

Utilizaram-se como mecanismo de busca os Descritores em Ciências da Saúde (DeCS) e Medical Subject Headings (MeSH): 'Health Care' ('Public Health'), 'Haiti' e 'Emigrants and Immigrants', combinados por meio do operador booleano AND, a fim de caracterizar a problemática da imigração de haitianos, no contexto dos cuidados e da assistência à saúde.

As buscas foram realizadas a partir das bases de dados Sistema Online de Busca e Análise de Literatura Médica (Medline/PubMed) e Scientific Electronic Library Online (SciELO), por dois pesquisadores, no mês de janeiro de 2020. Tais bases foram as únicas que apresentaram publicações disponíveis, a partir dos mecanismos de busca delimitados, considerando-se uma busca anterior no portal da Biblioteca Virtual em Saúde (BVS). Delimitaram-se como critérios de inclusão artigos publicados em periódicos científicos, no período de 2015-2019 (5 anos), disponíveis em português, inglês ou espanhol e que tratassem sobre a temática. Como critérios de exclusão, consideraram-se artigos duplicados nas bases, dissertações, teses e literatura cinzenta.

Para a busca na SciELO utilizou-se a seguinte combinação: 'Health Services' AND 'Haiti'. Na Medline/ PubMed, ‘Health Care' ('Public Health') AND ‘Haiti’ AND ‘Emigrants and Immigrants'. 


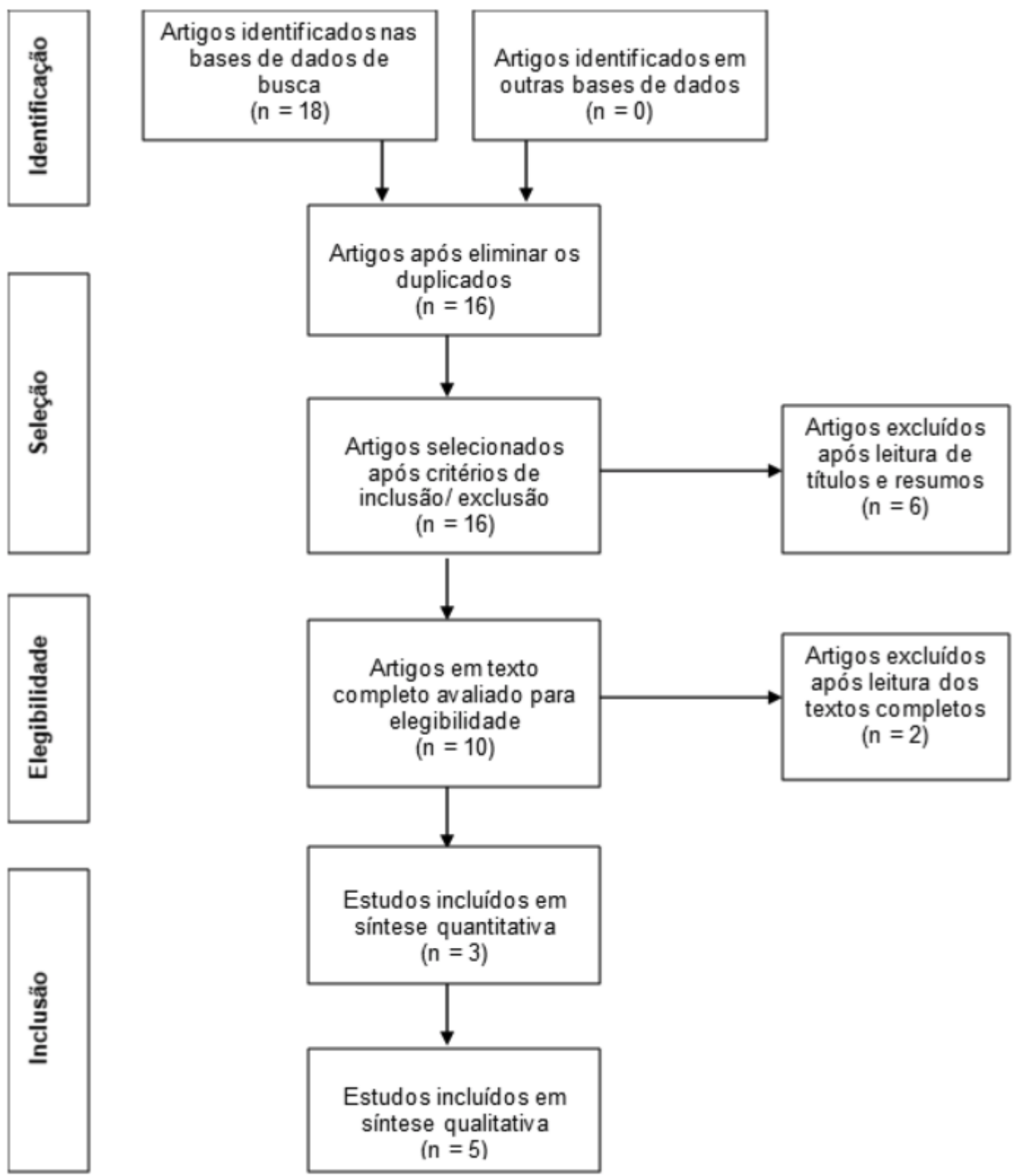

Figura 1 - Estratégias de busca dos artigos acerca da atenção à saúde de imigrantes haitianos no período de 2015 a 2019, incluídos na revisão. Três Lagoas, MS, Brasil (2020)

Fonte: elaborado pelos autores a partir de dados da pesquisa. 
A partir desses critérios, identificaram-se, inicialmente, 18 artigos nas bases consultadas, sendo 8 a partir da busca na SciELO e 10 na Medline/PubMed. Os artigos foram exportados para o gerenciador de referências EndNote e, após a exportação, excluíram-se os artigos duplicados nas bases, restando 16 artigos; em seguida, precedeu-se à análise dos títulos e resumos dos artigos, por dois revisores examinadores, buscando-se identificar a pertinência dos mesmos com a temática delimitada a partir da questão norteadora da pesquisa, sendo eliminados 6 artigos.

Posteriormente, os artigos foram lidos na íntegra e foi utilizado um instrumento para a coleta de dados, contendo os seguintes tópicos: título do artigo; título do periódico; ano da publicação; nomes dos autores e países de afiliação; contexto de realização do estudo (países, região); problemas e/ou situações de saúde investigados; principais achados; recomendações para a atenção à saúde de imigrantes haitianos. Após a leitura na íntegra, outros dois artigos foram excluídos por não estarem relacionados com a questão norteadora do estudo, sendo delimitado o corpus de oito artigos neste estudo.

\section{RESULTADOS}

Os resultados das buscas nas bases de dados envolvendo saúde e imigrantes resultaram em um grande número de publicações nos últimos cinco anos (1724). Contudo, as buscas envolvendo especificamente saúde de imigrantes haitianos demonstraram uma literatura ainda escassa no assunto, sendo identificados 16 artigos, dos quais apenas oito tratavam unicamente da problemática da saúde de imigrantes haitianos. 
Quadro 1 - Produção científica acerca da atenção à saúde de imigrantes haitianos no período de 2015 a 2019. Três Lagoas, MS, Brasil (2020)

\begin{tabular}{|c|c|c|c|c|c|c|}
\hline & Autor & Título & $\begin{array}{l}\text { Periódico e ano } \\
\text { de publicação }\end{array}$ & País & Objetivo & Tipo de estudo \\
\hline 1 & SÁNCHEZ et al. & $\begin{array}{l}\text { Haití, la nueva comunidad inmigrante en } \\
\text { Chile }\end{array}$ & $\begin{array}{l}\text { Revista Chilena de } \\
\text { Pediatría, } 2018\end{array}$ & Chile & $\begin{array}{l}\text { Conhecer a cultura haitiana e sua situação em saúde para uma } \\
\text { melhor compreensão de suas necessidades de saúde. }\end{array}$ & $\begin{array}{l}\text { Opinião, Relato de } \\
\text { experiência }\end{array}$ \\
\hline 2 & KEYS et al. & $\begin{array}{l}\text { Cholera control and anti-Haitian stigma in the } \\
\text { Dominican Republic: from migration policy to } \\
\text { lived experience }\end{array}$ & $\begin{array}{l}\text { Anthropology and } \\
\text { Medicine, } \\
2019\end{array}$ & \begin{tabular}{|l} 
Estados \\
Unidos
\end{tabular} & $\begin{array}{l}\text { Compreender o estigma e perspectivas etnográficas e políticas, } \\
\text { com base na experiência vivenciada em uma cultura local, bem } \\
\text { como uma análise da política de imigração e seu discurso. }\end{array}$ & $\begin{array}{l}\text { Estudo documental } \\
\text { e qualitativo }\end{array}$ \\
\hline 3 & BORGES et al. & $\begin{array}{l}\text { Socioeconomic and health profile of Haitian } \\
\text { immigrants in a Brazilian Amazon State }\end{array}$ & $\begin{array}{l}\text { Journal of } \\
\text { Immigrant and } \\
\text { Minority Health, } \\
2018\end{array}$ & Brasil & $\begin{array}{l}\text { Realizar uma análise da imigração haitiana por meio de } \\
\text { uma pesquisa realizada com as seguintes questões: jornada } \\
\text { migratória; características socioeconômicas e demográficas; } \\
\text { situação de saúde referida; estilo de vida; e acesso a cuidados } \\
\text { de saúde. }\end{array}$ & Estudo quantitativo \\
\hline 4 & ZUCHI et al. & $\begin{array}{l}\text { Screening and evaluation of lymphatic } \\
\text { filariasis in immigrants from endemic } \\
\text { countries residing in a focus where it is } \\
\text { considered eliminated in the Southern Region } \\
\text { of Brazil: a risk of reemergence? }\end{array}$ & $\begin{array}{l}\text { Acta Tropical, } \\
2017\end{array}$ & Brasil & $\begin{array}{l}\text { Avaliar a ocorrência de infecção filarial entre imigrantes } \\
\text { haitianos residentes na cidade de Chapecó, Santa Catarina, } \\
\text { por meio do Antígeno Filarial Circulante (AFC) para } \\
\text { Wuchereria bancrofti (Wb) realizado por 'point of care AD12- } \\
\text { immunochromatography card' (POC-ICT). }\end{array}$ & Estudo quantitativo \\
\hline 5 & PANG et al. & $\begin{array}{l}\text { Examining the association between } \\
\text { possessing a regular source of healthcare } \\
\text { and adherence with cancer screenings } \\
\text { among Haitian households in Little Haiti, } \\
\text { Miami-Dade County, Florida }\end{array}$ & $\begin{array}{l}\text { Medicine } \\
\text { (Baltimore), } 2017\end{array}$ & \begin{tabular}{|l} 
Estados \\
Unidos
\end{tabular} & $\begin{array}{l}\text { Avaliar a associação entre ter uma fonte regular de assistência } \\
\text { médica e a adesão aos exames de câncer recomendados na } \\
\text { população de Little Haiti em Miami. }\end{array}$ & $\begin{array}{l}\text { Estudo quantitativo, } \\
\text { documental, } \\
\text { retrospectivo }\end{array}$ \\
\hline 6 & MOISE et al. & $\begin{array}{l}\text { Diabetes knowledge, management, and } \\
\text { prevention among Haitian immigrants in } \\
\text { Philadelphia }\end{array}$ & $\begin{array}{l}\text { The Diabetes } \\
\text { Educator, } \\
2017\end{array}$ & \begin{tabular}{|l|} 
Estados \\
Unidos
\end{tabular} & $\begin{array}{l}\text { Gerar uma visão culturalmente enquadrada no conhecimento, } \\
\text { na gestão e prevenção da diabetes entre os imigrantes } \\
\text { haitianos em risco ou que estão atualmente vivendo com } \\
\text { diabetes tipo } 2 \text {. }\end{array}$ & $\begin{array}{l}\text { Estudo de natureza } \\
\text { qualitativa }\end{array}$ \\
\hline 7 & KENYA et al. & $\begin{array}{l}\text { Human papilloma virus and cervical cancer } \\
\text { education needs among HIV-positive Haitian } \\
\text { women in Miami }\end{array}$ & $\begin{array}{l}\text { Women's Health } \\
\text { Issues, } 2015\end{array}$ & \begin{tabular}{|l} 
Estados \\
Unidos
\end{tabular} & $\begin{array}{l}\text { Examinar o conhecimento sobre Papilomavírus Humano } \\
\text { (HPV)/câncer do colo do útero e identificar preferências de } \\
\text { intervenção entre mulheres haitianas HIV-positivas. }\end{array}$ & $\begin{array}{l}\text { Estudo de natureza } \\
\text { qualitativa }\end{array}$ \\
\hline 8 & $\begin{array}{l}\text { SANON; } \\
\text { SPIGNER; } \\
\text { MCCULLAGH. }\end{array}$ & $\begin{array}{l}\text { Transnationalism and hypertension self- } \\
\text { management among Haitian immigrants }\end{array}$ & $\begin{array}{l}\text { Journal of } \\
\text { Transcultural } \\
\text { Nursing, } \\
2016\end{array}$ & $\begin{array}{l}\text { Estados } \\
\text { Unidos }\end{array}$ & $\begin{array}{l}\text { Discutir a capacidade de gerenciamento da hipertensão em um } \\
\text { grupo de imigrantes haitianos relacionando tal capacidade com } \\
\text { o transnacionalismo. }\end{array}$ & $\begin{array}{l}\text { Estudo de natureza } \\
\text { qualitativa, } \\
\text { fundamentada } \\
\text { em referenciais } \\
\text { da etnografia } \\
\text { crítica e do } \\
\text { transnacionalismo }\end{array}$ \\
\hline
\end{tabular}

Fonte: elaborado pelos autores a partir de dados da pesquisa. 
Quadro 2 - Implicações práticas a partir dos resultados da produção científica acerca da atenção à saúde de imigrantes haitianos no período de 2015 a 2019. Três Lagoas, MS, Brasil (2020)

\section{Principais achados}

1 O impacto da migração existe, mas favorece aspectos positivos, como o efeito sobre a taxa de natalidade nacional reduzida, na incorporação à força de trabalho e na abertura para outras visões de mundo. Assim, embora com graus variados de dificuldade, o processo de integração está sendo realizado, o que favorece o acolhimento e a busca por medidas que facilitem o desempenho no trabalho, contribuindo significativamente para o cumprimento dos anseios da população nativa e dos imigrantes, além de atenuar a chamada Síndrome de Ulisses ou do emigrante com estresse crônico e múltiplo. dominicanos incorporou suas explicações no âmbito da responsabilidade individual, associando a cólera a supostas falhas de caráter entre os haitianos. Em contraste, os haitianos associaram os ataques cotidianos à violência estrutural, em especial travay mikwob (trabalho sujo), falta de acesso a água potável e a saneamento básico e interações interpessoais prejudiciais com os dominicanos.

3 Com a pesquisa foi possível encontrar um caso de migração impulsionada pela economia, mas para um cenário incomum. Concluímos que nossa população, em geral, mostrou boas condições de saúde, apesar da migração de um ambiente perigoso (terremoto, violência, pobreza). Em relação ao Sistema Único de Saúde (SUS), também foi possível ser constatada uma avaliação positiva, em que os cuidados necessários são prestados, mas cuja qualidade ainda precisa ser avaliada.

$4 \quad$ Com a pesquisa foi possível constatar que imigrantes haitianos infectados pela filariose residem nos municípios estudados de Santa Catarina e podem ser expostos a picadas do mosquito, levando à possibilidade de reemergência da filariose no estado. Postula-se, então, a necessidade de implementação de políticas locais de saúde pública com o objetivo de identificar imigrantes afetados, sendo tal medida crucial para manter as áreas livres da doença. Esforços devem ser feitos para desenvolver um inquérito internacional cooperativo entre países do Caribe que obtiveram sucesso na extinção da filariose (Suriname, Trindade e Tobago e Costa Rica) e países que estão em processo de controle e eliminação da doença (Guiana, Brasil, República Dominicana e Haiti), evitando o surgimento de filariose nesses países ou a persistência dos focos atuais.

\section{Implicações práticas}

A situação da imigração no Chile precisa passar de uma imigração livre, com fronteiras quase abertas, para a imigração regulamentada, por meio da esperada Lei de Migração, que permita o melhor enfrentamento dos impactos advindos do processo de imigração.

A partir do estudo, indica-se reformar o sistema de documentação, fortalecer o financiamento da saúde e restringir o estigma anti-haitiano nos níveis estadual e local. Essa responsabilidade não pode ser deixada somente a cargo do governo dominicano, devendo a população também se envolver.

Com base na pesquisa, é possível indicar que é necessária a realização de mais estudos longitudinais para avaliar melhor a questão da imigração haitiana. Estudos futuros também são necessários para avaliar a vulnerabilidade, a integração sociocultural e a saúde dos imigrantes haitianos no Brasil. Questões como violência durante as viagens migratórias, xenofobia e discriminação racial contra imigrantes também merecem atenção de estudiosos e atores sociais.

Pela análise dos dados, recomendam-se as seguintes atividades: 1) o treinamento de técnicos de laboratórios municipais e estaduais em coleta de amostras de sangue espesso e no POC-ICT (agora substituída pela Alere Filariasis Test Strip (FTS)); 2) seguir o protocolo emitido pelo Ministério da Saúde do Brasil, em casos positivos; 3) o treinamento dos trabalhadores do Programa Saúde da Família para identificar e aconselhar os imigrantes sobre a importância do diagnóstico da filariose linfática $(\mathrm{FL})$, com foco na preservação da saúde e das famílias; 4) sensibilizar e incentivar os migrantes de áreas endêmicas da FL a se submeter aos exames da FL. Ac̃ố os migrantes de areas endemicas da FL a sesucon como palestras, encontros em igrejas, associações, empresas que têm maiores
concentrações de trabalhadores e instituições que representam esses indivíduos são possíveis pontos de partida para essa orientação; 5) manter ativa a vigilância nas fronteiras do Brasil que apresentam o maior influxo de imigrantes; 6) os profissionais médicos em áreas não endêmicas precisam estar cientes e serem treinados para realizar o diagnostico diferencial entre a população imigrante proveniente de endêmicas da FL; e 7) divulgar os resultados negativos e positivos tratados e, apó triagem em laboratório, liberar seuś certificados de cura para todos os indivíduos investigados, utilizando o Sistema Único de Saúde (Ministério da Saúde). 


\section{Principais achados}

5 O foco do estudo foi em três exames preventivos: rastreamento do câncer colorretal, mamografias e exames de Papanicolaou. Após a pesquisa, verificou-se que os cuidados regulares estavam significativamente associados à triagem do câncer colorretal e à adesão à mamografia. $\mathrm{A}$ discrepância entre a mamografia e a adesão ao exame de Papanicolaou pode ser explicada pelo fato de que mamografias de rotina são necessárias com mais frequência, enquanto o exame de Papanicolaou tem um período maior de tempo entre as ocorrências.

O seguro desempenha um papel importante nos pacientes que obtêm os cuidados adequados dos quais precisam; com a maioria das triagens preventivas cobertas pelo seguro, os pacientes que possuem o plano podem ter maior probabilidade de aderir às triagens recomendadas, devido a uma redução nos custos diretos. Também se verificou forte associação entre ter uma fonte regular de cuidados e a maior adesão ao rastreamento do câncer colorretal e à mamografia.

6 Com a pesquisa, os indivíduos relataram diabetes e alto uso de insulina, complicações como fadiga, retinopatia, neuropatia e outros sinais de mau controle da doença também foram relatados. Notouse que pacientes com apoio familiar, em relação aos regimes alimentares, ao uso da medicação e aos registros da glicemia capilar, obtinham melhor controle da doença. Essa descoberta ressalta a oportunidade de utilizar a estrutura familiar para direcionar comportamentos em relação à doença.

7 A amostra da população estudada tinha conhecimento limitado sobre o HPV - câncer de colo de útero; equívocos sobre triagem, transmissão e tratamento eram comuns. Os participantes sentiram que o estigma dos prestadores impactou negativamente o atendimento recebido e o estigma da comunidade diminuiu o apoio social. Expressou-se o anseio por apoio a intervenções culturalmente adaptadas para melhorar o conhecimento sobre o HPV - câncer de colo de útero. Embora nenhum dos participantes tenha feito parte de pesquisas anteriormente, todos estavam dispostos a participar de ensaios futuros.

8 Derivaram-se da pesquisa dois grandes temas: apoio social e obrigação financeira, ambos ligados à questão monetária, pois surgiu uma dualidade em que o apoio social facilitou a autogestão da hipertensão pelos participantes da pesquisa, mas consequentemente representava um encargo financeiro para a parte apoiadora. 0 estudo evidenciou também que o transnacionalismo, embora influencie positivamente o bem-estar psicossocial dos imigrantes, pode afetar negativamente a experiência com o gerenciamento da doença. Ao cuidar desse grupo de imigrantes, os profissionais da saúde foram instigados a responder por esta interação transnacionalismo e gerenciamento de doenças. $O$ objetivo era contribuir para o entendimento dessa relação.

\section{Implicações práticas}

Verificou-se que são necessárias mais abordagens direcionadas para melhorar o acesso a cuidados regulares, o que pode melhorar a adesão ao rastreamento de câncer entre essa população imigrante.

Sugere-se que pesquisas futuras devam explorar o grau que os pacientes cumprem ou não as recomendações alimentares para prevenção e controle da diabetes; bem como se profissionais da saúde, como endocrinologistas, enfermeiros e nutricionistas, têm competência cultural para passar essas informações aos imigrantes. Mais pesquisas sobre esse assunto podem ser usadas visando o desenvolvimento de recomendações para prevenção de diabetes e ações de manejo com populações haitianas.

A pesquisa destaca a necessidade crítica de que a educação efetiva sobre HPV e câncer do colo de útero seja integrada aos serviços de saúde pública, atendendo haitianos, americanos e outras populações culturalmente distintas. A educação por pares ministrada pelo(s) ACS (Agente Comunitário de Saúde) foi identificada como um método culturalmente aceitável para melhorar o conhecimento da doença entre os participantes deste estudo.

Para ampliar a compreensão da relação do transnacionalismo com o processo saúde/doença, indica-se a realização de pesquisas sobre esse fenômeno com outras populações de imigrantes.

Fonte: elaborado pelos autores a partir de dados da pesquisa. 


\section{DISCUSSÃO}

A partir da análise dos artigos encontrados nesta revisão, identificaram-se três categorias de análise temática, as quais serão discutidas abaixo.

\section{Categoria 1: Impactos das regulamentações imigratórias na integração dos imigrantes haitianos}

Esta categoria descreve os impactos das regulamentações imigratórias na integração dos imigrantes haitianos, demonstrando que a falta de controle na entrada dos haitianos e as falhas nas legislações podem repercutir na falta de planejamento de ações voltadas para uma melhor integração dos mesmos no país receptor. Os artigos identificaram como impactos: estresse (SÁNCHEZ P, 2018) dos nativos e imigrantes; xenofobia (BORGES et al., 2018); discriminação (BORGES et al., 2018); estigma (KEYS et al., 2019); falta de qualidade no acesso a serviços de saúde (BORGES et al., 2018; ZUCHI et al., 2017; PANG et al., 2017; MOISE et al., 2015; KENYA et al., 2015); falta de acesso à água potável e ao saneamento básico (KEYS et al., 2019); dificuldades no financiamento da saúde (KEYS et al., 2019); doenças (KEYS et al., 2019; ZUCHI et al., 2017; PANG et al., 2017; MOISE et al., 2015; KENYA et al., 2015); falta de capacitação/formação profissional (ZUCHI et al., 2017; MOISE et al., 2015; KENYA et al., 2015).

Os artigos demonstraram que a ausência de regulamentações migratórias ou a inadequação das mesmas gera importantes impactos em todos os envolvidos, nativos e imigrantes.

Um estudo (PHILBIN; FLAKE; HATZENBUEHLER; HIRSCH, 2018) documental realizado nos EUA ressaltou que a imigração em nível estadual e as políticas voltadas aos imigrantes influenciam a saúde desse grupo populacional por meio de quatro caminhos, que incluem o racismo e o acesso a instituições, a serviços de saúde e a bens materiais. O estresse relacionado ao racismo estrutural impacta no acesso a instituições sociais benéficas, particularmente a educação e os serviços de saúde. No país, há preocupação em prover o acesso a condições materiais básicas, como alimentos, salários, trabalho e moradia.

Uma pesquisa (CALLAGHAN et al., 2019) americana qualitativa realizada com agentes comunitários de saúde identificou, por meio de grupos focais, que as mudanças na legislação migratória, decorrentes do governo Trump, têm proporcionado diferentes impactos na saúde de imigrantes, destacando-se: o crescente medo de interagir com os serviços sociais e de saúde, pelo receio da deportação; às barreiras de transporte para acessar os serviços de saúde devido ao aumento de pontos de fiscalização longe das áreas de fronteiras; as dificuldades significativas no acesso de crianças a programas nutricionais e a outros benefícios públicos.

Outro estudo (FLEMING et al., 2019) qualitativo realizado em Michigan, cujo objetivo foi entender como as modificações nas políticas pós-eleitorais podem ter impactado na saúde e no bem-estar, evidenciou mudanças na utilização de serviços sociais e de saúde por imigrantes. Também identificou impactos para a saúde física e mental desses indivíduos, relacionados ao medo crescente e generalizado de deportação e separação familiar. Aspectos que, por sua vez, resultaram em menor coesão comunitária, com influência negativa nos comportamentos relacionados à saúde de famílias.

Vale comentar os achados da pesquisa (INKOCHASAN et al., 2019) sobre os obstáculos legais que os migrantes enfrentam no acesso aos serviços de saúde nos países da sub-região do Grande Mekong, continente Asiático. A pesquisa abordou como tais obstáculos impactam na eliminação da malária e da resistência à artemisinina na região. A falta de legislação inclusiva para migrantes nesses países culminou em barreiras de acesso aos serviços de saúde para migrantes, como idioma e/ou fatores socioeconômicos.

Estudo (RUIZ-AZAROLA et al., 2018) qualitativo fenomenológico, conduzido em Andaluzia, Espanha, em duas fases (2009-2010 e 2012-2013), demonstrou diferenças no acesso dos migrantes à assistência médica, antes e depois da aplicação da Legislação do Decreto Real - RDL 16/2012, no qual foram instituídas 
medidas urgentes para garantir a sustentabilidade do Sistema Nacional de Saúde e melhorar a qualidade e a segurança dos serviços. Observou-se o agravamento de barreiras de acesso a serviços de saúde, após a implementação do decreto, tais como tempo de espera ou incompatibilidade de horários, além de piores condições socioeconômicas e administrativas dos participantes.

No Brasil, um estudo (VENTURA, 2018) apontou que a migração haitiana culminou na discussão sobre a aplicação do estatuto dos refugiados (Lei no 9.474/1997). Reitera-se que, além dos obstáculos legais relativos à documentação, mostram-se ausentes estratégias e políticas de acolhimento, o que resulta em atitudes discriminatórias e dificuldades de integração. A partir de um movimento de atores da sociedade civil, promulgou-se uma nova Lei de Migração no país, Lei nº 13.445, de 24 de maio de 2017 (BRASIL, 2017), que incorporou avanços importantes, imediatamente neutralizados e desvirtuados pelo Decreto Presidencial no 9.199, de 20 de novembro de 2017.

No contexto dos EUA, as desigualdades e barreiras encontradas no sistema de saúde são particularmente problemáticas para os imigrantes. Os imigrantes residentes permanentes só são elegíveis para programas de seguro público de saúde como Medicaid e CHIP após cinco anos no país, o que complica o acesso à saúde para imigrantes legais não cobertos por seguro privado patrocinado pelo empregador, responsável por mais da metade de todo o seguro no país. Nos EUA, imigrantes sem documentos não são elegíveis para nenhum programa de seguro de saúde administrado pelo governo (CALLAGHAN et al., 2019).

Desse modo, observa-se que as legislações voltadas à imigração nos países apresentam-se como importantes instrumentos para a garantia aos imigrantes de condições básicas de saúde ou, ao contrário, para o agravamento dessas condições e para a falta de acesso a serviços de saúde.

\title{
Categoria 2: competência intercultural para a atenção à saúde de imigrantes haitianos
}

Alguns dos artigos (MOISE et al., 2017; KENYA et al., 2015; SANON; SPIGNER; MCCULLAGH, 2016) encontrados nesta revisão enfatizaram a importância do desenvolvimento de competências interculturais e de sistemas de saúde culturalmente competentes para a atenção à saúde de imigrantes haitianos.

Define-se sistema de saúde culturalmente competente como:

\begin{abstract}
Aquele que reconhece e incorpora - em todos os níveis - a importância da cultura, a avaliação das relações interculturais, a vigilância em relação às dinâmicas resultantes de diferenças culturais, a expansão do conhecimento cultural e a adaptação de serviços para atender necessidades culturalmente únicas. Um sistema culturalmente competente também é construído com base na conscientização da integração e interação de crenças e comportamentos em saúde, prevalência eincidência de doenças e resultados de tratamentos para diferentes populações de pacientes (BETANCOURT; GREEN; CARRILLO; ANANEHFIREMPONG 2ND, 2003, p. 294).
\end{abstract}

A competência cultural abrange uma ampla gama de estratégias, que variam do nível individual ao nível político, e está incluída em inúmeros programas de treinamento para profissionais da saúde. Entre outros exemplos, intervenções culturalmente competentes podem incluir o desenvolvimento de serviços de saúde culturalmente específicos ou campanhas de promoção da saúde etnicamente sensíveis, o envio de intérpretes e mediadores interculturais em unidades de saúde, bem como políticas de saúde favoráveis aos migrantes (DAUVRIN; LORANT, 2015).

Contudo, para o desenvolvimento de competência intercultural para a atenção à saúde de imigrantes haitianos, faz-se necessário conhecer um pouco da sua história, cultura, dos seus costumes e acerca da organização da assistência à saúde no Haiti.

A estrutura social no Haiti é patriarcal, embora tenha havido um papel progressivo e maior projeção das mulheres, as quais desempenham o papel de dona de casa, sendo vistas como o pilar central da família. A religião é importante para a sociedade haitiana, pois contribui para conceder justiça e oferecer soluções 
de cura, influenciando o sistema educacional, especialmente as instituições católicas (SÁNCHEZ P et al., 2018).

Na religião católica predominante, existe uma coexistência e fusão com a prática do vodu. Na tradição do Haiti, contar histórias de origem africana, a música, a dança, a sopa tradicional de abóbora e uma veneração do sobrenatural são elementos fundamentais que compõem a expressão da identidade nacional. Seu sistema de saúde é formado por provedores dos setores público (35,7\%), privado (32,5\%), sem fins lucrativos mistos (31,8\%) e tradicional (SÁNCHEZ P et al., 2018).

Quase $80 \%$ de toda a população tem acesso às práticas de uma medicina complementar amplamente estendida, enquanto a medicina moderna é frequentemente o último recurso, especialmente para aqueles que vivem em setores rurais, a depender ainda de questões de educação e de crenças religiosas (SÁNCHEZ P et al., 2018).

Assim, a falta de desenvolvimento de competências interculturais nos profissionais de serviços de saúde pode dificultar o acesso dos imigrantes haitianos aos serviços e cuidados de saúde.

Um dos estudos (MOISE et al., 2017) identificados nesta revisão, com o objetivo de gerar uma visão culturalmente estruturada do conhecimento, gerenciamento e prevenção de diabetes entre os haitianos da Filadélfia (EUA), identificou, a partir da técnica de entrevista e grupos focais, que os médicos e enfermeiros são os profissionais mais envolvidos na transmissão de informações sobre a doença, devendo fornecer, inclusive, informações de saúde culturalmente competentes. Por exemplo, nutricionistas podem colaborar com pacientes de origens culturalmente diversas ao criarem um plano alimentar que acomode pratos culturais. O estudo também identificou a importância do apoio familiar na adesão ao tratamento, sendo esta uma oportunidade de utilizar a estrutura familiar para o direcionamento de comportamentos positivos à saúde.

Uma revisão sistemática de literatura (KLEIN; KNESEBECK, 2018), com o objetivo de avaliar as desigualdades na utilização de serviços de saúde entre migrantes e nativos na Alemanha, identificou uma menor utilização de serviços de saúde por migrantes para atendimento especializado, para uso de medicamentos, consultas e aconselhamento de terapeutas, reabilitação e prevenção de doenças (detecção precoce do câncer, programas de prevenção para crianças e exames de saúde bucal).

Para a maioria dos estudos extraídos na revisão, não foi possível descobrir os motivos da não utilização dos cuidados de saúde. Contudo, indica-se que barreiras linguísticas, falta de informações sobre o sistema de saúde e falta de competência intercultural dos membros das equipes de saúde são os principais motivos para a não utilização.

Um estudo (BRADY; VELJANOVA; SCHABRUN; CHIPCHASE, 2018) clínico randomizado conduzido em comunidades de imigrantes na Austrália, com o objetivo de avaliar o envolvimento do paciente e a viabilidade de uma nova abordagem de controle da dor, evidenciou que os participantes submetidos à fisioterapia culturalmente adaptada apresentaram maior adesão ao atendimento e tratamento. Demonstrou-se a importância de se alinhar o tratamento às crenças e aos valores de comunidades cultural e linguisticamente diversificadas para um melhor envolvimento do paciente e para resultados melhores à saúde dele.

Vale mencionar o estudo (HIGGINBOTTOM et al., 2015) de revisão sistemática que apresenta uma síntese narrativa dos achados de relatos de pesquisas empíricas a fim de fornecer perspectivas sobre os serviços de assistência à maternidade vivenciados por mulheres imigrantes no Canadá. Este estudo identificou que as mulheres imigrantes enfrentam muitas barreiras no acesso e na utilização desses serviços no país. Tais barreiras incluem falta de informação ou conscientização dos serviços, apoio insuficiente para acessar esses serviços e expectativas discordantes entre as mulheres e seus prestadores de serviços. Além disso, constatou-se que as diferenças culturais também causaram dificuldades para as mulheres imigrantes 
na compreensão das informações, e algumas se queixaram de que o cuidado à maternidade nem sempre é cultural e linguisticamente apropriado para elas.

\section{Categoria 3: condições de saúde e acesso a serviços por imigrantes haitianos}

Esta revisão demonstrou, a partir dos achados de alguns artigos (BORGES et al., 2018; ZUCHI et al., 2017; PANG et al., 2017; KENYA et al., 2015; SANON; SPIGNER; MCCULLAGH, 2016), que os imigrantes haitianos enfrentam dificuldades no acesso a serviços de saúde nos países em que se encontram e que tais dificuldades aumentam o risco de adquirirem condições e doenças debilitantes. A falta de acesso aos serviços, identificada a partir dos resultados dos estudos, encontra-se ligada a situações que impactam na qualidade desse acesso, principalmente no que tange ao acesso relacional. Assim, relações marcadas por discriminação, preconceito e falta de reconhecimento dos aspectos culturais e religiosos impactam no atendimento de qualidade, na conscientização dos haitianos sobre as medidas de prevenção e nos cuidados com a saúde e, consequentemente, no aumento da vulnerabilidade e de doenças para eles.

Destaca-se, a partir do editorial publicado pelo periódico PLOS Medicine (TURNER, 2019), que trabalhadores migrantes que se mudaram internacionalmente estão sob risco de lesões profissionais, estão vulneráveis a surtos graves de doenças infecciosas (como a cólera) e a doenças de seu país de origem (como tuberculose), além do risco de doenças não transmissíveis. Os problemas de saúde mental, incluindo o transtorno de estresse pós-traumático, também são uma preocupação particular para migrantes e refugiados em muitos contextos. Além disso, tal como discutido nas categorias anteriores, barreiras de idioma, cultura ou lei impedem que os migrantes acessem os serviços essenciais de saúde.

A condição de saúde dos imigrantes é um aspecto central para a inserção e integração deles à sociedade. Exige compreender o processo de saúde, de doença e cuidado com esses grupos, bem como refletir sobre as respectivas responsabilidades dos estados. Alguns desafios são apontados: a sustentabilidade dos sistemas de saúde nacionais; o efetivo acesso à atenção integral de saúde de nacionais e imigrantes; os meios e recursos adequados para o enfrentamento das doenças transmissíveis e não transmissíveis de impacto local e mundial; a captação e alocação de recursos para pesquisa; e um desenvolvimento científico e tecnológico que atenda à maioria da população mundial (VENTURA, 2018).

Tais desafios foram identificados na revisão (HAYWARD; HARDING; MCSHANE; TANNER, 2018) sobre influências biológicas e antropológicas relacionadas ao risco de exposição a patógenos, à vulnerabilidade a infecções ou desenvolvimento de doenças ativas, bem como o acesso a tratamento por minorias étnicas e migrantes no Reino Unido. O estudo ressaltou ser um equívoco comum a compreensão de que os migrantes tenham uma incidência maior de tuberculose em comparação com a população em geral, simplesmente porque a 'importam' do exterior. Evidenciou-se que, além da associação com a residência pré-migração em países de alta incidência, outros fatores influenciam, tais como: suscetibilidade genética; deficiência de vitamina $\mathrm{D}$, devido a fatores climáticos e alimentares; comorbidades; privação socioeconômica; fatores relacionados à experiência da migração; barreiras de acesso ao tratamento, à saúde; diferenças culturais nos comportamentos de busca de tratamento; e estigmatização de pacientes.

Outro estudo (PANG et al., 2017) verificou que a população imigrante haitiana sofre muitas disparidades de saúde que criam barreiras para a obtenção de exames preventivos no Condado de Miami-Dade, Flórida.

Os transtornos mentais também parecem afetar frequentemente as populações imigrantes. Entretanto, uma revisão sistemática de literatura (FOO et al., 2018) não encontrou evidências conclusivas de um aumento no risco de depressão ou de outros transtornos do humor nos membros da comunidade migrante.

Uma explicação para esse resultado encontra-se no conceito de resiliência, um construto que representa adaptação positiva, competência social e psicológica em tempos de adversidades ou traumas significativos. É possível que os migrantes possuam fatores de resiliência não compartilhados com os nativos, o que 
explica por que nenhum risco diferencial de resultados mentais adversos foi observado entre os dois grupos (FOO et al., 2018).

Todavia, um estudo (BETANCOURT; GREEN; CARRILLO; ANANEH-FIREMPONG 2ND, 2003) ressaltou que, em situações nas quais alguns membros da família são deportados e outros permanecem no país, evidencia-se um aumento de problemas de saúde mental associados, os quais podem ser resolvidos por meio da identificação das origens da angústia, elucidando estruturas e papéis familiares, fortalecendo práticas de comunicação, vinculando-se a recursos legais e econômicos.

As condições de trabalho de imigrantes também estão relacionadas ao surgimento de problemas de saúde, tal como identificado em um estudo (LEÃO et al., 2017) com o objetivo de caracterizar a população de imigrantes haitianos em Cuiabá e Várzea Grande, Mato Grosso, Brasil. Os resultados da pesquisa mostraram uma precária situação de trabalho dos imigrantes haitianos, sendo os riscos físicos e de acidentes os mais percebidos, como também foram relatadas manifestações de sofrimento físico e psicossocial.

Por fim, o transnacionalismo foi explorado em um estudo (SANON; SPIGNER; MCCULLAGH, 2016) identificado nesta revisão, enquanto impacto negativo no gerenciamento da doença por parte dos imigrantes haitianos. Os transmigrantes são indivíduos que sustentam relações múltiplas e criam identidades transnacionais, mantendo um relacionamento próximo com amigos e familiares em seu país de origem, enquanto se engajam ativamente nos assuntos do seu país anfitrião. Apesar dessa rede de apoio, o estudo revela que tais identidades, que motivam a formação de laços entre os imigrantes, estão relacionadas a aspectos negativos, como uma maior pressão por envio de recursos financeiros para familiares. Essa conjuntura pode influenciar nocivamente comportamentos em relação ao manejo da doença. Um estudo (SILVA, 2019) ressaltou que a experiência transnacional torna-se natural no processo migratório, mesmo que este resulte em dificuldades, tal como a aceitação da violência como um processo natural.

\section{CONCLUSÃO}

Os resultados desta revisão indicam que a população imigrante haitiana lida com diferentes barreiras de acesso no cuidado com a saúde e que está sujeita a diferentes situações de vulnerabilidade em todos os países incluídos nas pesquisas e avaliados nesta revisão.

Tais condições de saúde, bem como o desenvolvimento de alguns problemas de saúde e manejo inadequado de doenças, podem ser potencializadas por legislações voltadas ao combate da imigração, ausência de políticas públicas de saúde direcionadas a essa população e falta de competência intercultural para a abordagem da mesma.

Apesar das limitações deste estudo, que incluem o pequeno número de artigos identificados nas bases e a não consideração de outros idiomas nativos dos haitianos, tais como o francês, os resultados desta revisão integrativa salientam a importância de se reconhecer os efeitos estressantes no processo de migração dos haitianos para o Brasil no que se refere ao cuidado em saúde. Nesse caso, destaca-se a regulamentação a que os imigrantes estão sujeitos para entrada nos países, inclusive relacionada à saúde, como a necessidade de seguro-saúde.

Ressalta-se também a importância de se considerar a competência intercultural para a atenção à saúde de imigrantes na formulação de políticas públicas de saúde, inclusive as políticas de formação em saúde. É fundamental rever nos currículos dos cursos de graduação da área de saúde a abordagem intercultural. Destaca-se também a importância de os gestores dos sistemas de saúde investirem na formação continuada dos seus profissionais para o desenvolvimento de competências interculturais, considerando-se as particularidades do modo de vida e da cultura haitiana.

Além de investimentos em formação de profissionais, torna-se necessário que os países invistam em políticas públicas voltadas às necessidades sociais e de saúde dos imigrantes haitianos. Deve-se aumentar 
a conscientização da população nativa com relação ao acolhimento dos imigrantes, evitando-se o estigma e o preconceito. Para a formulação de políticas públicas de saúde, é preciso considerar os aspectos culturais, as crenças e a importância do apoio social que se faz presente no transnacionalismo.

\section{REFERÊNCIAS}

BETANCOURT, Joseph R.; GREEN, Alexander R.; CARRILLO, J. Emilio; ANANEH-FIREMPONG 2ND, O. Defining cultural competence: a practical framework for addressing racial/ethnic disparities in health and health care. Public Health Reports (Washington, D.C.: 1974), [Washington, D.C], v. 118, n. 4, p. 293-302, 2003. DOI: https://doi.org/10.1093/phr/118.4.293. Disponível em: https://pubmed.ncbi.nlm.nih.gov/12815076/. Acesso em: 08 mar. 2020.

BISPO, Glaucia Margarida Bezerra et al. Avaliação do acesso de primeiro contato na perspectiva dos profissionais. Revista Brasileira de Enfermagem, Brasília, v. 73, n. 3, e20180863, 2020. DOI: https:// doi.org/10.1590/0034-7167-2018-0863. Disponível em: https://www.scielo.br/scielo.php?script=sci arttext\&pid=S0034-71672020000300173\&tlng=en. Acesso em: 10 maio 2020.

BORGES, Fabiano Tonaco et al. Socioeconomic and health profile of Haitian immigrants in a Brazilian Amazon State. Journal of Immigrant and Minority Health, [Nova lorque], v. 20, n. 6, p. 1373-1379, 2018. DOI: https:// doi.org/10.1007/s10903-018-0694-9. Disponível em: https://link.springer.com/article/10.1007/s10903-018-06949. Acesso em: 08 mar. 2020.

BRADY, Bernadette; VELJANOVA, Irena; SCHABRUN, Siobhan; CHIPCHASE, Lucinda. Integrating culturally informed approaches into physiotherapy assessment and treatment of chronic pain: a pilot randomised controlled trial. BMJ Open. [s. I.], v. 8, n. 7, e021999, 2018. DOI: https://doi.org/10.1097/ nnr.0000000000000092. Disponível em: https://pubmed.ncbi.nlm.nih.gov/25871625/. Acesso em: 08 mar. 2020.

BRASIL. Lei No. 13.445, de 24 de maio de 2017. Institui a Lei de Migração. Diário Oficial da União: seção 1, Brasília, DF, n. 99, p. 1-2, 25 maio 2020. Disponível em: http://www.planalto.gov.br/ccivil 03/ Ato20152018/2017/Lei/L13445.htm. Acesso em: 08 mar. 2020.

BRZOZOWSKI, Jan. Migração internacional e desenvolvimento econômico. Estudos Avançados, São Paulo, v. 26, n. 75, p. 137-156, ago. 2012. DOI: https://dx.doi.org/10.1590/S0103-40142012000200009. Disponível em: https://www.scielo.br/scielo.php?script=sci_arttext\&pid=S0103-40142012000200009\&lng=pt\&tlng=pt. Acesso em: 10 maio 2020.

CALLAGHAN, T et al. Immigrant health access in Texas: policy, rhetoric, and fear in the Trump era. BMC Health Services Research, [s. I.], v. 19, n. 1, p. 342, 2019. DOI: https://doi.org/10.1186/s12913-019-4167-1. Disponível em: https://bmchealthservres.biomedcentral.com/articles/10.1186/s12913-019-4167-1 Acesso em: 08 mar. 2020.

DAUVRIN, Marie; LORANT, Vincent. Leadership and cultural competence of healthcare professionals: a social network analysis. Nursing research, [Nova lorque], v. 64, n. 3, p. 200-210, 2015. DOI: https://doi.org/10.1097/ nnr.0000000000000092. Disponível em: https://pubmed.ncbi.nlm.nih.gov/25871625. Acesso em: 08 mar. 2020.

ESCUDERO, Camila. A voz da mulher imigrante no debate público sobre o "Projeto pró-cesárea no SUS" em São Paulo a partir da perspectiva da comunicação intercultural. Revista Eletrônica de Comunicação, Informação e Inovação em Saúde, Rio de Janeiro, v. 13, n. 4, p. 736-753, out.-dez 2019. DOI: http://dx.doi. org/10.29397/reciis.v13i4.1850. Disponível em: https://www.reciis.icict.fiocruz.br/index.php/reciis/article/ view/1850. Acesso em: 10 maio 2020.

FAQUETI, Amanda; GRISOTTI, Marcia; RISSON, Ana Paula. Saúde de imigrantes haitianos: revisão de estudos empíricos qualitativos. Interface - Comunicação, Saúde, Educação, Botucatu, v. 24 p. e190311, 2017. DOI: https://doi.org/10.1590/interface.190311. Disponível em: https://www.scielo.br/scielo. php?script=sci_arttext\&pid=S1414-32832020000100301. Acesso em: 08 mar. 2020.

FLEMING, Paul J. et al. A qualitative study on the impact of the 2016 US election on the health of immigrant families in Southeast Michigan. BMC Public Health, [Londres], v. 19, n. 1, p. 947, 2019. DOI: https://doi. org/10.1186/s12889-019-7290-3. Disponível em: https://pubmed.ncbi.nlm.nih.gov/31307435/. Acesso em: 08 mar. 2020.

FOO, Shea Q. et al. Prevalence of depression among migrants: a systematic review and meta-analysis. International Journal of Environmental Research and Public Health, Basel, v. 15, n. 9, p. 1986, 2018. DOI: 
https://doi.org/10.3390/ijerph15091986. Disponível em: https://pubmed.ncbi.nlm.nih.gov/30213071/. Acesso em: 08 mar. 2020.

GRANADA, Daniel; CARRENO, Ioná; RAMOS, Natália; RAMOS, Maria da Conceição Pereira. Discutir saúde e imigração no contexto atual de intensa mobilidade humana. Interface - Comunicação, Saúde, Educação, Botucatu, v. 21, n. 61, p. 285-296, 2017. DOI: https://doi.org/10.1590/1807-57622016.0626. Disponível em: https://www.scielo.br/scielo.php?script=sci_arttext\&pid=S1414-32832017000200285\&lng=pt\&tlng=pt. Acesso em: 10 maio 2020.

GUERRA, Katia; VENTURA, Miriam. Bioética, imigração e assistência à saúde: tensões e convergências sobre o direito humano à saúde no Brasil na integração regional dos países. Cadernos Saúde Coletiva, Rio de Janeiro, v. 25, n. 1, p. 123-129, 2017. DOI: https://doi.org/10.1590/1414-462x201700010185. Disponível em: https://www.scielo.br/scielo.php?script=sci_arttext\&pid=S1414-462X2017000100123\&lng=en\&nrm=iso\&tln $\mathrm{g}=\mathrm{pt}$. Acesso em: 08 mar. 2020.

HAYWARD, Sally; HARDING, Rosaline M.; MCSHANE, Hellen; TANNER, Rachel. Factors influencing the higher incidence of tuberculosis among migrants and ethnic minorities in the UK [version 2; peer review: 2 approved]. F1000 Research, [s. I.], v. 7, p. 461, c2018. Preprint. DOI: https://dx.doi.org/10.12688\%2Ff1000res earch.14476.2. Disponível em: https://f1000research.com/articles/7-461/v2. Acesso em: 08 mar. 2020.

HIGGINBOTTOM, Gina MA et al. Immigrant women's experiences of maternity-care services in Canada: a systematic review using a narrative synthesis. Systematic Reviews, [Londres], v. 4, p. 13, 2015. DOI: https://dx.doi.org/10.1186\%2F2046-4053-4-13. Disponivel em: https://www.ncbi.nlm.nih.gov/pmc/articles/ PMC4506414/. Acesso em: 08 mar. 2020.

INKOCHASAN, Montira et al. Access to health care for migrants in the Greater Mekong Subregion: policies and legal frameworks and their impact on malaria control in the context of malaria elimination. WHO SouthEast Asia Journal of Public Health, [Nova Deli], v. 8, n. 1, p. 26-34, 2019. DOI: https://doi.org/10.4103/22243151.255346. Disponível em: https://pubmed.ncbi.nlm.nih.gov/30950427l. Acesso em: 08 mar. 2020.

KENYA, Sonjia et al. Human papilloma virus and cervical cancer education needs among HIV-positive Haitian women in Miami. Women's Health Issues, [Nova lorque], v. 25, n. 3, p. 262-266, 2015. DOI: https://doi. org/10.1016/j.whi.2014.12.007. Disponível em: https://pubmed.ncbi.nlm.nih.gov/25864021/. Acesso em: 08 mar. 2020.

KEYS, Hunter M. et al. Cholera control and anti-Haitian stigma in the Dominican Republic: from migration policy to lived experience. Anthropology and Medicine, [Abingdon], v. 26, n. 2, p. 123-141, 2019. DOI: https://doi.org/10.1080/13648470.2017.1368829. Disponível em: https://www.scielo.br/scielo.php?script=sci arttext\&pid=S0104-07072008000400018. Acesso em: 08 mar. 2020.

KLEIN, Jens; KNESEBECK, Olaf von dem. Inequalities in health care utilization among migrants and nonmigrants in Germany: a systematic review. International Journal for Equity in Health, [Londres], v. 17, n. 1, p. 160, 2018. DOI: https://dx.doi.org/10.1186\%2Fs12939-018-0876-z. Disponível em: https://www.ncbi.nlm.nih. gov/pmc/articles/PMC6211605/. Acesso em: 08 mar. 2020.

LEÃO, Luís Henrique da Costa et al. Migração internacional, saúde e trabalho: uma análise sobre os haitianos em Mato Grosso, Brasil. Cadernos de Saúde Pública, Rio de Janeiro, v. 33, n. 7, e00181816, 2017. DOI: https://doi.org/10.1590/0102-311x00181816. Disponível em: https://www.scielo.br/scielo.php?script=sci arttext\&pid=S0102-311X2017000706001\&lng=en\&nrm=iso\&tlng=pt. Acesso em: 08 mar. 2020.

MENDES, Karina Dal Sasso; SILVEIRA, Renata Cristina de Campos; GALVÃO, Cristina Maria. Revisão integrativa: método de pesquisa para a incorporação de evidências na saúde e na enfermagem.

Texto \& Contexto - Enfermagem, Florianópolis, v. 17, n. 4, p. 758-764, 2008. DOI: https://doi. org/10.1590/S0104-07072008000400018. Disponível em: https://www.scielo.br/scielo.php?script=sci arttext\&pid=S0104-07072008000400018. Acesso em: 08 mar. 2020. 
MOISE, Rhoda K. et al. Diabetes knowledge, management, and prevention among Haitian immigrants in Philadelphia. The Diabetes Educator. [Thousand Oaks], v. 43, n. 4, p. 341-347, 2017. DOI: https://doi. org/10.1177/0145721717715418. Disponível em: https://pubmed.ncbi.nlm.nih.gov/28627321/. Acesso em: 08 mar. 2020.

OLIVEIRA, Helena Nobre de; SILVA, César Augusto Marques da; OLIVEIRA, Antonio Tadeu Ribeiro de. Imigração internacional: uma alternativa para os impactos das mudanças demográficas no Brasil? Revista Brasileira de Estudos de População, [s. I.], v. 36, p. 1-31, 2019. DOI: https://doi.org/10.20947/s01023098a0076. Disponível em: https://www.rebep.org.br/revista/article/view/1254. Acesso em: 10 maio 2020.

PANG, Hauchie et al. Examining the association between possessing a regular source of healthcare and adherence with cancer screenings among Haitian households in Little Haiti, Miami-Dade County, Florida. Medicine (Baltimore). [Hagerstown], v. 96, n. 32, e7706, 2017. DOI: https://doi.org/10.1097/ md.0000000000007706. Disponível em: https://pubmed.ncbi.nlm.nih.gov/28796056/. Acesso em: 08 mar. 2020.

PHILBIN, Morgan M.; FLAKE, Morgan; HATZENBUEHLER, Mark L.; HIRSCH, Jennifer S. State-level immigration and immigrant-focused policies as drivers of Latino health disparities in the United States. Social Science \& Medicine (1982), [Nova lorque], v. 199, p. 29-38, 2018. DOI: https://doi.org/10.1016/j. socscimed.2017.04.007. Disponível em: https://pubmed.ncbi.nlm.nih.gov/28410759/ Acesso em: 08 mar. 2020.

RISSON, Ana Paula; MATSUE, Regina Yoshie; LIMA, Ana Cristina Costa. Atenção em saúde aos imigrantes haitianos em Chapecó e suas dimensões étnico-raciais. O Social em Questão, Rio de Janeiro, v. 31, n. 41, p. 111-130, 2018. Disponível em: http://osocialemquestao.ser.puc-rio.br/media/OSQ_41_art_5_Risson_Matsue Lima.pdf. Acesso em: 08 mar. 2020.

RUIZ-AZAROLA, Ainhola et al. La perspectiva de personas migrantes sobre el acceso a la atención sanitaria en el contexto de políticas de austeridad en Andalucía. The perspective of migrants on access to health care in the context of austerity policies in Andalusia (Spain). Gaceta Sanitaria, Barcelona, v. 34, n. 3, p. 261267, 2018. DOI: http://dx.doi.org/10.1016/j.gaceta.2018.09.006. Disponível em: http://scielo.isciii.es/scielo. php?script=sci abstract\&pid=S0213-91112020000300009\&Ing=es\&nrm=iso\&tlng=en. Acesso em: 08 mar. 2020.

SÁNCHEZ P, Katheryn et al. Haití, la nueva comunidad inmigrante en Chile. Revista Chilena de Pediatría, Santiago, v. 89, n. 2, p. 278-283, 2018. DOI: http://dx.doi.org/10.4067/S0370-41062018000200278. Disponível em: https://scielo.conicyt.cl/scielo.php?script=sci_arttext\&pid=S0370-41062018000200278. Acesso em: 08 mar. 2020.

SANON, Marie-Anne; SPIGNER, Clarence; MCCULLAGH, Marjorie C. Transnationalism and hypertension self-management among Haitian immigrants. Journal of Transcultural Nursing, [Memphis], v. 27, n. 2, p. 147-156, 2016. DOI: https://doi.org/10.1177/1043659614543476. Disponível em: https://pubmed.ncbi.nlm.nih. gov/25062700/. Acesso em: 08 mar. 2020.

SANTOS, Fabiene Vinente. A inclusão dos migrantes internacionais nas políticas do sistema de saúde brasileiro: o caso dos haitianos no Amazonas. História, Ciências, Saúde-Manguinhos, Rio de Janeiro, v. 23, n. 2, p. 477-494, 2016. DOI: https://doi.org/10.1590/S0104-59702016000200008. Disponível em: https://www. scielo.br/scielo.php?script=sci_arttext\&pid=S0104-59702016000200477\&lng=pt\&tlng=pt. Acesso em: 10 maio 2020.

SILVA, Lucas Rech da. Educação e migração haitiana: um estudo de caso a partir de Galtung e Fanon. Educação por Escrito, Porto Alegre, v. 10, n. 2, e36671, 2019. DOI: https://doi.org/10.15448/21798435.2019.2.36671. Disponível em: https://revistaseletronicas.pucrs.br/ojs/index.php/porescrito/article/ view/36671. Acesso em: 10 maio 2020.

SOUZA, Marcela Tavares de; SILVA, Michelly Dias da; CARVALHO, Rachel de. Revisão integrativa: o que é e como fazer? Einstein (São Paulo), São Paulo, v. 8, n. 1, p. 102-106, 2010. DOI: https://doi.org/10.1590/ s1679-45082010rw1134. Disponível em: https://www.scielo.br/scielo.php?script=sci arttext\&pid=S167945082010000100102\&lng=en\&nrm=iso\&tlng=pt. Acesso em: 10 maio 2020. 
TURNER, Richard. Migrants and refugees: improving health and well-being in a world on the move. PLOS Medicine, São Francisco, v. 16, n. 7, e1002876, 2019. DOI: https://doi.org/10.1371/journal.pmed.1002876. Disponível em: https://journals.plos.org/plosmedicine/article?id=10.1371/journal.pmed.1002876. Acesso em: 08 mar. 2020.

URQUIZA, Antônio Hilário Aguilera; RIBEIRO, Leonardo Cavallini. Direitos Humanos e migração: os paradoxos da globalização. Argumenta Journal Law, Jacarezinho, n. 28, p. 217-239, 2018. DOI: http://dx.doi. org/10.35356/argumenta.v0i28.1188. Disponível em: http://seer.uenp.edu.br/index.php/argumenta/article/ view/1188. Acesso em: 10 maio 2020.

VENTURA, Miriam. Immigration, global health, and human rights. Cadernos de Saúde Pública, Rio de Janeiro, v. 34, n. 4, e00054118, 2018. DOI: https://doi.org/10.1590/0102-311x00054118. Disponível em: https:// www.scielo.br/scielo.php?script=sci_arttext\&pid=S0102-311X2018000400201\&lng=pt\&tlng=pt. Acesso em: 08 mar. 2020.

ZUCHI, Arthur et al. Screening and evaluation of lymphatic filariasis in immigrants from endemic countries residing in a focus where it is considered eliminated in the Southern Region of Brazil: a risk of reemergence? Acta Tropica, [Amsterdan], v. 176, p. 192-196, 2017. DOI: https://doi.org/10.1016/j.actatropica.2017.08.010. Disponível em: https://pubmed.ncbi.nlm.nih.gov/28823910. Acesso em: 08 mar. 2020. 